Tatiana Retinskaya*

\title{
CELLULE XIII DE JEAN ROGISSART : FUSION DES ÉLÉMENTS DU FRANÇAIS HORS NORME ${ }^{1}$
}

\section{INTRODUCTION}

L'étude du fonds argotique et dialectal d'une langue nationale peut être réalisée selon deux axes : stylistique et lexicologique. Pour notre recherche, nous avons choisi la voie stylistique qui est fondée sur l'analyse des éléments non conventionnels incorporés dans les textes littéraires des auteurs régionaux.

Il est à indiquer que les unités non conventionnelles constituent une partie importante du roman régional. La plupart des œuvres des maîtres du roman régional sont marquées par la suprématie des sources du français local. L'emploi des lexèmes populaires, argotiques et/ou familiers est plutôt un moyen sporadique utilisé par les écrivains régionalistes. Néanmoins, un des romans de Jean Rogissart ${ }^{2}$ (1894-1961), auteur ardennais, se distingue des textes créés par des romanciers symbolisant tel ou tel terroir de l'Hexagone.

Dans le cadre de cet article, nous proposons la description du substrat non codifié dans le roman Cellule XIII, substrat composé de deux couches lexicales qui ne peuvent pas être attribuées au français standard. L'utilisation de la première couche du vocabulaire spécifique - des mots localement limités - s'explique par «l'ancrage régional $»^{3}$ de Jean Rogissart. Quant au recours aux argotismes qui représentent la deuxième couche du répertoire lexical étudié, il convient de remarquer que l'auteur cède la place primordiale des idiomes ardennais aux unités appartenant plutôt à l'argot traditionnel pour relever le statut social de ses héros et leur conception du monde. Par ailleurs, dans le titre, nous employons le mot « fusion » pour souligner l'importance de deux sous-systèmes de la langue dans la narration et la nécessité de l'insertion des mots en question pour identifier les locuteurs et leur époque.

La sélection de cette œuvre littéraire est également liée au thème générique du $\mathrm{X}^{\mathrm{e}}$ Colloque international d'Argotologie « Comment parle-t-on de la guerre en termes populaires / argotiques ». En effet, la guerre est présente dans le roman du début à la fin.

\footnotetext{
* tatiana.retinskaya@yahoo.fr
}

1 L'étude est effectuée dans le cadre du projet scientifique № 16-04-50078 soutenu par la Fondation russe pour la recherche fondamentale.

2 Je tiens à remercier M. Michel Tamine de m'avoir fourni des ouvrages et des documents qui m'ont permis de découvrir et d'étudier l'œuvre de Jean Rogissart.

3 Pour cet écrivain ardennais, " l'ancrage régional constitue avant tout l'ascèse qui permet à l'écriture de se développer pleinement » (Tamine 2011a: 4). 


\section{PRÉSENTATION DU ROMAN CELLULE XIII}

Le roman Cellule XIII est le septième tome du cycle des Mamert édité en 1961, l'année de la mort de Jean Rogissart. Ce dernier roman, que l'auteur n'a pas eu « le plaisir ni d'ouvrir en connaisseur, ni de dédicacer à ses amis » (Manceau 1984 : 547), est dans une grande mesure un texte autobiographique. Comme Jean Rogissart, Michel Mamert, personnage principal, est un instituteur dans les Ardennes et subit une arrestation par défaut lors de l'occupation nazie de son département :

Une chose importante, c'est qu'il a été emprisonné un mois en 1943. On n'a jamais su exactement pourquoi. J'ai moi-même été emprisonné de juillet à août 43, pour une histoire de carte d'identité. [...] Rogissart a été emprisonné à ce moment-là. [...] De ma cellule, je voyais Rogissart dans la cour, mais nous ne pouvions pas nous parler (Tamine 2011b : 20).

L'auteur situe les événements décrits dans le roman pendant l'année 1944 ; année qui suit celle de son incarcération. Cette dernière partie de la « vaste fresque retraçant sur plus d'un siècle (1830-1944) et à travers l'histoire d'une famille, l'évolution sociale, économique, politique de la zone la plus industrialisée de la vallée de la Meuse » (Tamine 2011c : 40), raconte comment la guerre peut non seulement changer brusquement le cours des choses, mais aussi ravager la vie de la famille dont les membres sont liés par des relations affectueuses et un soutien mutuel.

\section{MODÈLE DE L'ANALYSE DU CORPUS}

L'algorithme de l'analyse de la source non conventionnelle du langage pour créer divers effets stylistiques a été élaboré par Éda Beregovskaya (1929-2011) lors de l'examen de cent quarante textes littéraires de plus de cent vingt écrivains français, édités dans les années 1945-1975. La fondatrice de l'École de Stylistique de Smolensk a consacré dix ans (de 1969 à 1979) à ce travail. Son modèle comprend les composantes ci-dessous :

1) la concentration des argotismes dans le texte;

2) la fréquence des unités argotiques ;

3) les formes de la sémantisation des argotismes, qui peuvent être extratextuelles (glossaire en appendice, note en bas de page), textuelles (glossaire en situation, traduction de l'argotisme par son analogue normatif et du mot normatif par l'argotisme, bref commentaire, interprétation détaillée, éclaircissement à l'aide d'une périphrase, emploi parallèle d'un synonyme neutre) et contextuelles ou « méthode directe »;

4) les frontières du «bloc argotique », défini comme une masse, à première vue non structurée, des argotismes répartis dans le texte à des intervalles variés et réunis par leur fonction stylistique commune dans l'œuvre ;

5) les fonctions essentielles du bloc argotique, telles que la création de l'ambiance émotionnelle générale, la typification du discours du personnage, la présentation de l'intégration du personnage à un nouveau milieu social, l'individualisation du discours du personnage, la mise en évidence de certains traits de son portrait 
moral, la transposition comique du sujet, la création du portrait grotesque (« travesti »), l'expression du degré de francisation d'un personnage non français, la représentation conventionnelle d'un argot étranger, la caractérisation concise d'un personnage ( « croquis »), la stylisation ou la mise en relief d'une situation ;

6) les possibilités expressives des argotismes ayant une valeur stylistique autonome : amplification de l'expression de différentes émotions, accentuation de l'incompréhension réciproque des personnages, fonctionnement en tant que mot de passe, création de l'effet de choc, organisation du jeu de mots.

Trois méthodes (descriptive, analytique, comparative), ainsi que celle de la statistique symptomatique, permettent d'étudier en détail l'effet stylistique des éléments non conventionnels et de déterminer la tendance dominante de leur utilisation (Beregovskaya 2009 : 52-98).

En utilisant l'algorithme particulier de l'analyse d'un texte littéraire contenant des lexèmes argotiques, nous comprenons que le choix expert et précis des argotismes, la manipulation de leurs formes et de leurs significations sont les conditions d'une description fiable des événements et de la conduite des personnages. Souvent le roman se transforme en confession et les romanciers, en révélant leur position sociale, invitent le lecteur à apprécier non seulement la description de la vie de leurs héros, mais aussi leur point de vue littéraire et moral. L'application du schéma du déchiffrement des unités non conventionnelles facilite le repérage des motifs de la création langagière et montre que l'argot devient un refuge où les argotiers peuvent exprimer leurs pensées et leurs sentiments et se faire plaisir en jouant avec le langage.

Il est nécessaire de mentionner que le modèle de l'analyse polyvalente du substrat hors norme élaboré par Éda Beregovskaya est applicable à la description des argotismes et des régionalismes dans le tissu du texte littéraire. Nous l'avons déjà mis en œuvre antérieurement avec succès. Notamment, il nous a permis de caractériser des éléments du français régional d'origine dialectale dans les œuvres des auteurs ardennais et champenois Joseph Cressot, Jean Rogissart et Yves Gibeau ${ }^{4}$. Il a été également utilisé pour le décodage des dialectismes dans Récits d'un chasseur d'Ivan Tourgueniev.

\section{LE RÔLE DES RÉGIONALISMES DANS LE ROMAN RÉGIONAL}

L'identification des traits particuliers du comportement verbal se réalise, entre autres, par la voie du substrat local.

L'analyse pragmatico-stylistique des régionalismes dans le texte littéraire ainsi que la description comparative des unités des français régionaux utilisées par des auteurs du terroir est l'un des procédés de l'étude de la régionalisation de la langue nationale. Le régiolecte qu'on peut caractériser comme « le résultat de la collision de deux tendances : celles de l'unification et de la différenciation » (Gak 1986 : 125) représente un macrosystème hétérogène.

4 Citons à titre d'exemple l'article « Le vocabulaire régiolectal d'Yves Gibeau (à partir de l'exemple du roman Mourir idiot)» (Retinskaya 2016). 
C'est au moyen de l'ensemble des lexèmes du parler local qui restent une des constantes considérables du roman régional que les auteurs du terroir réussissent à graver les traits les plus typiques de leurs compatriotes et de cette façon permettre de sauvegarder le patrimoine linguistique.

Dans cette partie de l'article, nous proposons de décrire les moyens de l'incorporation et de l'explication textuelle des régionalismes utilisés par le maitre du roman ardennais jouant le rôle de décrypteur des particularités des unités localement limitées et faisant fonction d'intermédiaire de la représentation de la vision du monde du locuteur.

La caractérisation de la concentration des régionalismes - première étape de l'analyse des sources non conventionnelles - montre que l'auteur utilise des mots locaux en faible proportion ${ }^{5}$. L'auteur a recours aux formes régionales non seulement pour dévoiler les particularités langagières des habitants ardennais, mais aussi pour manifester son amour pour sa patrie; de sorte que l'emploi de termes de l'aire natale devient une mission majeure d'un écrivain régional. Et il en est bien ainsi de l'auteur de Cellule $X I I I$. En effet, comment « Jean Rogissart pouvait-il peindre l'Ardenne sans les mots d'Ardenne ?» (Tamine $1994: 11$ ).

L'une des premières tournures locales employées par Jean Rogissart est chardon Rolland: "Mais pourquoi revoir aussi le bon monsieur Cayasse, professeur émérite, qui avait pu cultiver sur des souches de chardon Rolland - des chardons roulants comme disaient les gens de son village de retraité » (Rogissart 1984 : 411). L'auteur fait référence à un idiome particulier à l'aide de la précision « comme disaient les gens de son village » qui constitue un procédé répandu du décodage textuel des régionalismes.

Si on examine la fréquence des moyens pour insérer des éléments du français régional des Ardennes dans le tissu du roman, on constate qu'une partie considérable des mots typiques est intégrée sans aucun commentaire ${ }^{6}$ :

Bê-là, ça leur apprendra, aux Mamert, de s'occuper d'autre chose que de leur métier. Trop payés pour ce qu'ils font ! bê-là, oui (Rogissart $1984: 421)$ (bê-là : exclamation pour acquiescer).

Nous, on en était tout couquis (Rogissart 1984 : 435) (couqui 'très étonné').

Notre pauvre maire en était flache comme une flatte ; il avait beau lever les bras, dire qu'ils n'y comprenait rien de rien, que jamais de sa vie il n'avait eu de fusil, ni de guerre, ni de chasse [...] (Rogissart 1984 : 435) (flatte 'bouse de vache').

5 Éda Beregovskaya (2009: 78) a relevé quatre types de concentration des unités hors norme dans les œuvres littéraires : forte concentration : 1 mot non conventionnel pour 10 à 100 mots, concentration moyenne (1:100 à 500), faible concentration (1:500 à 1000), concentration insignifiante ( $1: 1000$ et plus).

6 C'est la méthode directe de l'introduction du lexique hors norme.

7 L'analyse des régionalismes est réalisée à la base des ouvrages lexicographiques de Michel Tamine/Robert Cecconello (1994), de Michel Tamine (2006) et d'Albert Vauchelet (1979). 
J'ai bien cru que ça n'en finirait jamais. À qui était le plus estomaqué de nous ou faisait cranse de l'être (Rogissart 1984 : 436) (faire cranse 'faire semblant').

Moi qui ne venais que pour faire quatrième à la belote, par bon cœur. J'aurais mieux fait de m'amuser avec les donzelles [...] (Rogissart 1984 : 436) (donzelle 'jeune fille').

Par la suite évincé de toutes les usines, il avait créé une coopérative d'alimentation et une Maison du Peuple. La guerre l'en avait chassé ; puis Léonce est né, culot de la bande (Rogissart 1984 : 442) (culot 'dernier né d'une famille').

Il m'a parlé de vous, de son grand-père et du ratayon Jean. Les Mamert, c'est comme une chaîne, un sillon bien droit et continu (Rogissart 1984 : 449) (ratayon 'ancêtre').

De nombreux exemples tirés des œuvres des auteurs régionalistes prouvent que « l'archaïsme linguistique rejoint le français dialectal ou régional » (Tamine $2011: 52$ ). Il en est ainsi du verbe choir 'tomber', vieilli en français, mais qui reste régionalement employé, et que Jean Rogissart introduit directement : « Et v'là t'y pas que nos quatre Verts rappliquent, furibards, harponnent le maire et le traînent au jardin derrière sa maison, si bien que le pauvre vieux qui a dans les soixante ans manque d'en choir » (Rogissart $1984: 435$ ).

L'incorporation de certaines formes régionales dont le degré de survivance est assez faible est effectuée au moyen de l'utilisation de synonymes appartenant à divers registres du français. Illustrons ce procédé de la sémantisation par la citation du microcontexte contenant le mot spécifique raulage 'vieille chose ou vieil objet sans valeur, mais que l'on met de côté car il est susceptible d'être récupéré' : Ça casse, ça brise tout et partout, pour trouver quoi ? des nippes, des frusques, des raulages de l'ancien temps, des vieilleries hord d'usage, mais nix fusils, nix ! (Rogissart 1984 : 434). Notons que l'auteur a mis le mot régional ci-dessus en italique pour attirer l'attention du lecteur.

L'analyse des mots et des expressions du langage local montre que le régiolecte a pour fonction pragmatique de révéler l'importance de la forme régionale de la langue nationale et du territoire sur lequel elle fonctionne. Les résultats de cette étude vont aider à mettre en lumière les facteurs extra- et intralinguistiques exerçant une influence sur la vitalité des régionalismes et à caractériser l'état actuel des modifications territoriales du français standard.

\section{LE RÔLE DES ARGOTISMES DANS LE ROMAN DE JEAN ROGISSART CELLULE XIII}

Les unités de l'argot traditionnel choisies par l'auteur contribuent à restituer l'atmosphère d'emprisonnement et à évoquer les jours passés en prison par le héros principal. Le vocabulaire argotique est attaché à la composition de l'œuvre : il fonctionne en bloc 
et correspond aux particularités langagières des détenus et à leur appartenance à certaines couches sociales. Le terrain privilégié des unités argotiques regroupées en bloc est le discours direct. Le tableau ci-dessous représente les argotismes les plus fréquents dans le roman étudié :

Tableau 1: Argotismes fréquents tirés du roman de Jean Rogissart Cellule XIII

\begin{tabular}{|l|c|}
\hline Argotisme & Nombre d'occurrences \\
\hline Chleuh 'soldat allemand' & 11 \\
\hline Fritz 'soldat allemand' & 3 \\
\hline patelin 'village' & 3 \\
\hline piaule 'chambre' & 3 \\
\hline salaud 'individu ignoble' & 3 \\
\hline schlague 'fouet' & 3 \\
\hline taule 'prison' & 3 \\
\hline Verts (Vert-de-gris) 'soldat allemand' & 3 \\
\hline dare-dare 'à toute vitesse' & 2 \\
\hline déguster 'souffrir' & 2 \\
\hline Doryphore 'soldat allemand' & 2 \\
\hline ficher, se 'se moquer' & 2 \\
\hline flingot 'fusil de guerre' & 2 \\
\hline jaser 'babiller sans arrêt' & 2 \\
\hline jus 'eau' & 2 \\
\hline mouchard 'indicateur de police' & 2 \\
\hline quille 'libération' & 2 \\
\hline trique 'expulsion' & 2 \\
\hline
\end{tabular}

Presque toutes les unités argotiques énumérées peuvent être qualifiées comme argotismes thématiques (terme d'Éda Beregovskaya) : leur désignation correspond à la thématique de l'œuvre. Certains argotismes employés une seule fois sont également relatifs aux sujets clefs de la narration : celui de la guerre et de l'incarcération par l'ennemi. Citons quelques-uns de ces lexèmes mentionnés : arroser 'mitrailler', se bouffer le nez (se tabasser) 'se battre', chiourme 'gardien', crever (claboter) 'mourir', dégonflard 'individu peu courageux', fourrer (coffrer) 'encarcérer', gnon 'coup', harponner 'arrêter', $r a b$ 'reste de vivres', vaches 'délateurs'.

Dans la plupart des cas, les dominantes sémantiques comportent des lexèmes qui représentent des centres d'attraction synonymique. Le rang synonymique « soldat allemand » (dont la dominante sémantique est « ennemi ») est un des plus révélateurs. On trouve ainsi : Chleuhs - Brutes vertes - Doryphores - Fridolins - Frisés - Fritz - Souris grises - Verts - Verts-de-gris.

À ce titre, il est des plus intéressants et symptomatiques que Jean Rogissart ait regroupé et organisé les mots sur des fiches intitulées selon les sujets à développer ; comme l'explique Michel Tamine, à propos - par exemple - de la fiche «Peur» : 
On peut imaginer que lorsqu'il recherchait un terme porteur d'une nuance particulière, il avait toutes chances de le trouver en se reportant à l'une de ses fiches. On trouve ainsi une fiche intitulée peur, riche d'une bonne soixantaine de formes non glosées mais regroupées par catégories grammaticales, et parfois, à l'intérieur d'une catégorie, par registres de langue, à l'instar de mots argotiques d'ailleurs soulignés en rouge (Tamine 2011c : 54).

En procédant à l'analyse des traits particuliers du bloc argotique créé par l'auteur ardennais, on a pu constater la dominance de quatre fonctions : 1) typification du discours du personnage, 2) présentation de l'intégration du personnage à un nouveau milieu social, 3) caractérisation concise d'un personnage et 4) mise en relief d'une situation.

Quant aux argotismes autonomes, on peut remarquer que ces éléments du français hors norme servent à identifier les initiés, aident à exprimer différentes émotions et accentuent la compréhension réciproque des personnages.

\section{LE CROISEMENT DES UNITÉS RÉGIONALES ET ARGOTIQUES}

La combinaison des régionalismes et des argotismes dans de nombreux microcontextes permet avant tout d'identifier l'utilisateur du régiolecte, plus particulièrement ses origines, son niveau d'enseignement, ses goûts, son savoir-vivre. À titre d'exemples, on peut relever plusieurs occurrences d'emploi parallèle de mots non conventionnels :

Avè c'te lavasse, on crèvera, oui (Rogissart 1984 : 450) (avè 'avec', lavasse 'breuvage insipide', crever 'mourir')!

Ah què kasbah, mes aminches, què taule (Rogissart 1984 : 454) (kasbah 'maison', aminche 'ami', taule 'prison') !

Vous avez entendu cette nuit ? Là, là. I dégustent, hein ? Què giboulée (Rogissart 1984 : 515) (què 'quelle', déguster 'souffrir')!

Il est important de souligner que les marqueurs diatopiques et diastratiques signalent au lecteur l'information qui facilitera la compréhension des conceptions de l'auteur.

\section{CONCLUSION}

L'analyse de deux segments du substrat non codifié incorporés dans le roman de l'auteur régionaliste montre que cette stratégie de l'utilisation des indices diatopiques et diastratiques s'avère juste et s'explique par la nécessité de l'identification des locuteurs et celle d'une caractérisation plus précise des événements.

C'est parce qu'elle est imprégnée de traits très spécifiques d'un langage régional appartenant à une aire linguistique circonscrite que cette œuvre contribue à la conservation du patrimoine linguistique. La description des termes argotiques utilisés par le maître du roman ardennais qui en a été faite au moyen d'outils explicatifs textuels 
a permis de montrer l'importance incontournable du statut social de l'utilisateur du régiolecte, ainsi que les constantes de sa perception du monde.

\section{Bibliographie}

BEREGOVSKAYA, Éda (2009) Argo i jazik sovremennoj franczuzskoj prozy. [L'argot et la langue de la prose française du XX' siècle]. Smolensk : Éds de l'Université d'État de Smolensk.

GAK, Vladimir (1986) Vvedenije vo franczuzskuju filologiju. [Introduction à la philologie française]. Moscou : Éds Prosveščenije.

MANCEAU, Henri (1984) « Jean Rogissart (1894-1961). » In : J. Rogissart, Les Mamert, 542-547.

RETINSKAYA, Tatiana (2016) « Regiolekntnij slovar’ Iva Žibo (na primere romana Mourir idiot). [Le vocabulaire régiolectal d'Yves Gibeau (à partir de l'exemple du roman Mourir idiot)]. » Les Cahiers de l'Université d'État d'Orel 4 (73), 195-199.

ROGISSART, Jean (1984) « Cellule XIII. » In : J. Rogissart, Les Mamert, 402-529.

ROGISSART, Jean (1984) Les Mamert. Vol. 2. Charleville-Mézières : Éds Terres Ardennaises.

TAMINE, Michel (1994) « Préface. » In : J. Rogissart, Les Romans rustiques. I. Charleville-Mézières : Éds Terres Ardennaises, 7-12.

TAMINE, Michel/Robert CECCONELLO (1994) « Lexique. » In : J. Rogissart, Les Romans rustiques. I. Charleville-Mézières : Éds Terres Ardennaises, 877-895.

TAMINE, Michel (2006) Le parler des Ardennes. Paris : Christine Bonneton Éditeur.

TAMINE, Michel (2011a) « Actualité de Jean Rogissart?» Terres Ardennaises. Revue d'histoire et de géographie locales 116, 3-4.

TAMINE, Michel (2011b) « Rogissart par ceux qui l'ont connu : témoignage d'André Patureaux. Interview.» Terres Ardennaises. Revue d'histoire et de géographie locales 116, 19-20.

TAMINE, Michel (2011c) « La langue de Rogissart : aspects lexicaux. » Terres Ardennaises. Revue d'histoire et de géographie locales 116, 40-57.

VAUCHELET, Albert (1979) Tous les patois des Ardennes. Vieux langages et vieilles choses. Charleville-Mézières : Imprimerie SOPAIC - Savreux.

\section{Résumé \\ CELLULE XIII DE JEAN ROGISSART : FUSION DES ÉLÉMENTS DU FRAN- ÇAIS HORS NORME}

Les unités hors norme constituent une partie importante du roman régional. Ce sont des régionalismes qui dominent les éléments du vocabulaire en question. Cependant le roman de Jean Rogissart Cellule XIII se distingue considérablement des textes des écrivains régionalistes. Dans le cadre de ce travail, nous proposons l'analyse de deux segments du substrat non codifié ci-dessous : 1) des formes répandues dans une aire ; 2) 
des unités appartenant à l'argot traditionnel. Il est à noter que le titre de notre article contenant le mot « fusion » montre l'importance de deux sous-systèmes de la langue dans la narration : l'insertion des lexèmes localement limités ainsi que des argotismes dans le tissu du texte est nécessaire pour l'identification des locuteurs et la caractérisation plus précise des événements.

L'auteur ardennais qui a consacré son dernier roman à la guerre a réussi non seulement à décrire la souffrance physique et morale subie par le peuple, mais aussi à graver les traits les plus typiques du langage de ses compatriotes et de cette façon à sauvegarder le patrimoine linguistique. L'étude des termes régionaux et argotiques utilisés par le maître du roman ardennais et des outils de leur explication textuelle permet de décrypter les particularités du parler localement limité et de présenter le statut social de l'utilisateur du régiolecte et sa vision du monde.

Mots-clés : régionalisme, argotisme, parler des Ardennes, roman regional, Jean Rogissart

\section{Abstract \\ CELLULE XIII BY JEAN ROGISSART: THE FUSION OF ELEMENTS OF NON-STANDARD FRENCH}

Nonconventional lexical units have an important place in the regional novel, and regionalisms dominate among the elements of the marked vocabulary. However, the novel Cellule XIII by Jean Rogissart is considerably different from the works of other regionalist writers. In this study, we propose an analysis of the two following segments of a nonconventional lexical units: 1) local lexemes; 2) lexemes of traditional argot. It is worth noting that the title of the article, which contains the word "fusion", shows the importance of two language subsystems in the narrative: the insertion of territorially restricted units and argotisms into the fabric of a literary text is necessary to identify the speaker and give a more accurate description of events.

Rogissart was an Ardennes author who dealt with the theme of war and managed not only to describe the physical and moral sufferings of the people, but also to convey the characteristic features of their compatriots, and thus preserve their linguistic heritage. The study of Rogissart's regional and argotic vocabulary and of its actual use in the novel makes it possible to also see how certain features serve to characterize the linguistic social status of a regional speaker and his or her worldview.

Keywords: regionalism, argotism, Ardennes regiolect, regional novel, Jean Rogissart 


\section{Povzetek \\ CELICA XII PISATELJA JEANA ROGISSARTA: SPAJANJE ELEMENTOV NENORMIRANE FRANCOŠČINE}

Nestandardne leksikalne enote igrajo pomemben vlogo $\mathrm{v}$ romanu, pisanem $\mathrm{v}$ regionalnem jeziku. V pričujočem besedišču prevladujejo »lokalizmi«. Roman Celica XIII Jeana Rogissarta se pomembneje razlikuje od ostalih besedil pisateljev, ki pišejo v dialektih. V okviru pričujoče raziskave podajamo analizo dveh segmentov substrata: (1) oblik, ki se pojavljajo na določenem območju; (2) enot, ki so del tradicionalnega argoja. Beseda »spajanje«, ki je del naslova, kaže na pomembnost obeh podsistemov jezika pripovedi: vstavljanje narečnih leksemov in argojevskih besed v besedilo je potrebno za prepoznavanje govorcev in natančnejšo opredelitev dogodkov.

Ardenski pisatelj Jean Rogissart, ki je svoj zadnji roman posvetil vojni, je uspel opisati ne samo fizično in moralno trpljenje ljudi, ampak tudi podati najbolj značilne poteze jezika svojih sorojakov in na ta način pripevati k ohranitvi jezikovne dediščine. Raziskava narečnih in argojevskih besed, ki se pojavljajo v besedilu romana, in način, kako so predstavljane, omogoči odkriti posebnosti dialektalno omejenega govora in predstaviti družbeni status uporabnika dialekta in njegovo videnje sveta.

Ključne besede: dialekt, argo, govor Ardenov, regionalni roman, Jean Rogissart 